\title{
Prognostic Significance of the pN Classification Supplemented by Vascular Invasion for Esophageal Squamous Cell Carcinoma
}

\author{
Chong-Mei Zhu ${ }^{1,29}$, Yi-Hong Ling ${ }^{1,29}$, Shao-Yan $\mathrm{Xi}^{1,2}$, Rong-Zhen Luo ${ }^{1,2}$, Jie-Wei Chen ${ }^{1,2}$, \\ Jing-Ping Yun ${ }^{1,2}$, Dan Xie ${ }^{1,2}$, Mu-Yan Cai ${ }^{1,2 *}$
}

1 Sun Yat-sen University Cancer Center, State Key Laboratory of Oncology in South China, Collaborative Innovation Center for Cancer Medicine, Guangzhou, China, 2 Department of Pathology, Sun Yat-sen University Cancer Center, Guangzhou, China

\begin{abstract}
Background: The biological behavior and clinical outcome of esophageal squamous cell carcinoma (ESCC) are difficult to predict.

Methodology/Principal Findings: We investigate the prognostic impact of vascular invasion to establish a risk stratification model to predict recurrence and overall survival. We retrospectively evaluated the vascular invasion of 433 patients with ESCC treated with surgery between 2000 and 2007 at a single academic center. Those patients were assigned to a testing cohort and a validation cohort by random number generated in computer. The presence of vascular invasion was observed in 113 of $216(52.3 \%)$ and 96 of $217(44.2 \%)$ of ESCC in the training and validation cohorts, respectively. Further correlation analysis demonstrated that vascular invasion in ESCC was significantly correlated with more advanced pN classification and stage in both cohorts $(P<0.05)$. Additionally, presence of vascular invasion in ESCC patients was associated closely with poor overall and recurrence-free survival as evidenced by univariate and multivariate analysis in both cohorts $(P<0.05)$. In the subset of ESCC patients without lymph node metastasis, vascular invasion was evaluated as a prognostic predictor as well $(P<0.05)$. More importantly, the combined prognostic model with $\mathrm{pN}$ classification supplemented by vascular invasion can significantly stratify the risk (low, intermediate and high) for overall survival and recurrence-free survival in both cohorts $(P<$ 0.05). The C-index to the combined model showed improved predictive ability when compared to the pN classification ( 0.785 vs 0.739 and 0.689 vs 0.650 for the training and validation cohorts, respectively; $P<0.05$ ).
\end{abstract}

Conclusions/Significance: The examination of vascular invasion could be used as an additional effective instrument in identifying those ESCC patients at increased risk of tumor progression. The proposed new prognostic model with the pN classification supplemented by vascular invasion might improve the ability to discriminate ESCC patients' outcome.

Citation: Zhu C-M, Ling Y-H, Xi S-Y, Luo R-Z, Chen J-W, et al. (2014) Prognostic Significance of the pN Classification Supplemented by Vascular Invasion for Esophageal Squamous Cell Carcinoma. PLOS ONE 9(4): e96129. doi:10.1371/journal.pone.0096129

Editor: Xin-Yuan Guan, The University of Hong Kong, China

Received February 17, 2014; Accepted April 2, 2014; Published April 24, 2014

Copyright: $\odot 2014$ Zhu et al. This is an open-access article distributed under the terms of the Creative Commons Attribution License, which permits unrestricted use, distribution, and reproduction in any medium, provided the original author and source are credited.

Funding: This study was supported by grants from the Nature Science Foundation of China (No. 81302139), the Foundation for Distinguished Young Talents in Higher Education of Guangdong (No. 84000-3211701) and the Program for Excellent Young Talents in Sun Yat-sen University Cancer Center (No. 520101210101). The funders had no role in study design, data collection and analysis, decision to publish, or preparation of the manuscript.

Competing Interests: The authors have declared that no competing interests exist.

*E-mail: caimuyan@hotmail.com

9 These authors contributed equally to this work.

\section{Introduction}

Esophageal cancer is the eighth most common cancer and the sixth leading cause of cancer death worldwide [1]. As the dominant type of esophageal cancer in China, esophageal squamous cell carcinoma (ESCG) distributes a general poor prognosis due to lack of a singular effective clinical method for early diagnosis. Despite improvements in its detection and treatment, the outcome in patients with ESCG remains poor, with an overall 5-year survival of $15-34 \%$ [2-3]. Most patients who undergo curative treatment for ESCG will eventually relapse and die as a result of this cancer. Given the poor outcome of ESCC and its high incidence, it is increasingly important to understand the progression of this cancer and to identify the most associated prognostic factors.

Appropriate risk stratified selection for adjuvant treatment trials is paramount, considering the high cost and toxic side effects of chemotherapeutic drugs. Different clinicopathological parameters such as tumor location, size, differentiation, infiltrative depth, lymph node involvement and distant metastasis, have been proposed as relevant factors to predict cancer-specific survival in patients with ESCC [4]. To improve the predictive accuracy of single prognosticator, the $7^{\text {th }}$ edition AJCC/IUAC TNM classification system comprising several clinicopathological features and predicting different outcomes have been constructed [5]. Although currently proposed TNM system shows considerable prognostic 


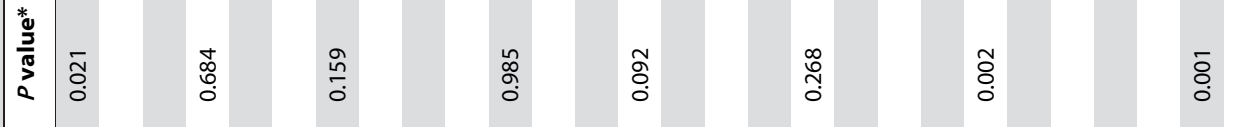

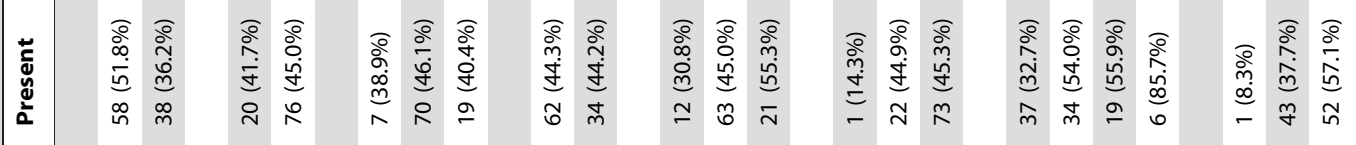

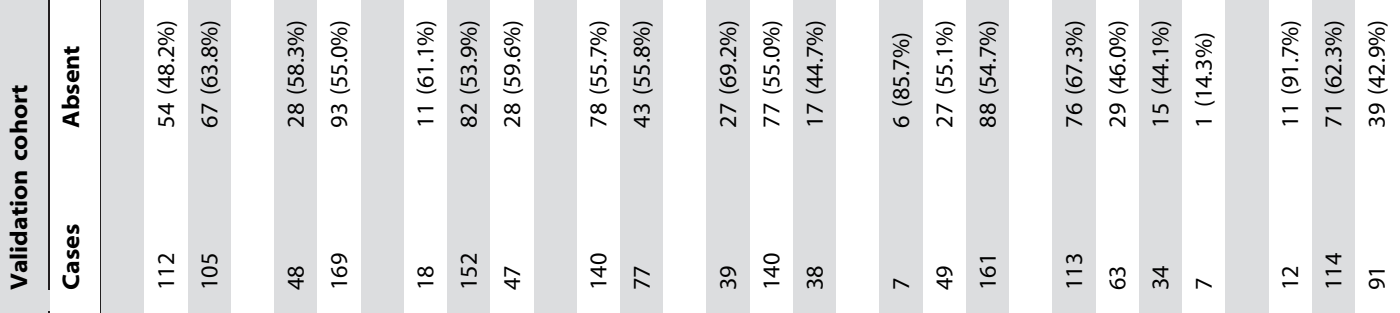

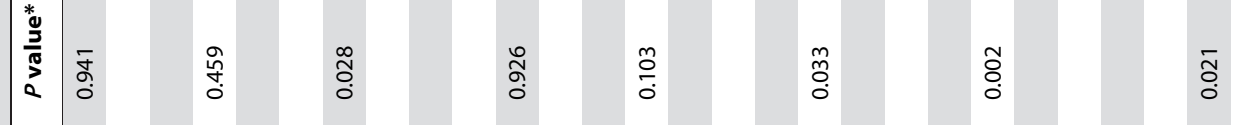

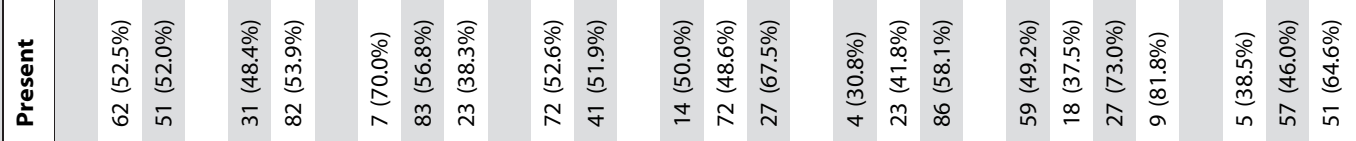

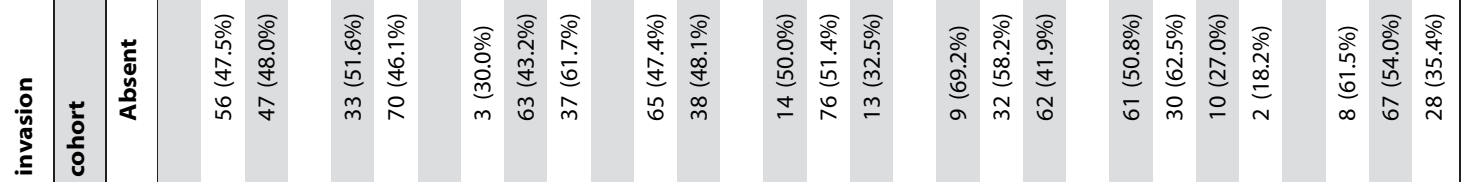

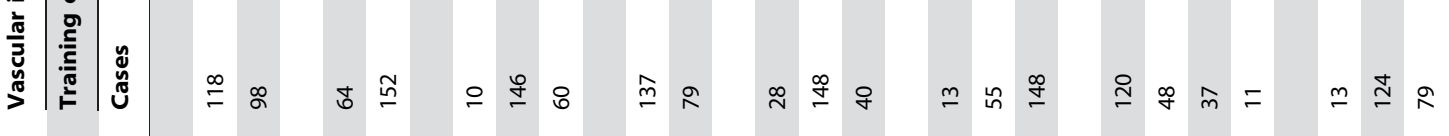


accuracy, there remains demand for increasing the accuracy of existing outcome predictive system.

Vascular invasion has been incorporated into TNM Classification of Malignant Tumors and College of American Pathologists Consensus Statement in pathological reports [6-8]. Assessment of vascular invasion with $\mathrm{H} \& \mathrm{E}$ staining enables identifying patients with high risk within the same TNM stage and therapeutic strategy can be tailored accordingly [9]. Since the report was investigated by Suqimachi et al. 1986 [10], numerous studies have been conducted on vascular invasion in ESCC. Most of these studies indicated that vascular invasion in ESCG was the relevant predictor of cancer-specific survival [4,11-13]. Additionally, vascular invasion in superficial ESCG is also found to be a strong risk factor correlated with lymph node metastasis [14]. Therefore, it seems to be an attractive prognostic predictor. However, several clinicopathological features failed to improve the accuracy of existing multivariate prognostic models when they were analyzed for their added value [15-16].

The objective of the current study was to assess the prognostic value of vascular invasion in patients with ESCG, focusing on the predictive significance of the $\mathrm{pN}$ classification supplemented by vascular invasion in a large, Chinese, single center cohort of patients with ESCG.

\section{Materials and Methods}

\section{Ethics Statement}

The study was approved by the Institute Research Medical Ethics Committee of Sun Yat-sen University. No informed consent (written or verbal) was obtained for use of retrospective tissue samples from the patients within this study, most of whom were deceased, since this was not deemed necessary by the Ethics Committee, who waived the need for consent. All samples were anonymised.

\section{Patients and Cohorts}

A total of 433 patients with ESCG, who underwent curative esophagectomy between October 2000 and May 2007, were randomly selected from the Department of Pathology of Sun Yatsen University Cancer Center (Guangzhou, China). The selective criteria were: (1) having no adjuvant treatment before operation; (2) complete resection of the tumor; (3) incised margin was negative; (4) without distant metastasis; (5) follow-up data was detailed and complete. Those patients were assigned to a testing cohort and a validation cohort by random number generated in computer.

The training cohort was composed of $152(70 \%)$ male and 64 $(30 \%)$ female, with median age of 56.5 years. Average follow-up time was 41.3 months (median, 38.5 months; range, 1.0-115.0 months). In parallel, the validation cohort included $169(78 \%)$ male and $48(22 \%)$ female, with a median age of 57.0 years. Average duration of follow-up in this cohort was 42.3 months (median, 39.0 months; range, 2.0-106.0 months).

We collected clinicopathologic data including patient age, gender, tumor location, tumor size, differentiation, TNM stage, infiltrative depth, lymph node status, vascular invasion and recurrence. These data are detailed in Table 1. Tumor differentiation was determined based on the criteria proposed by WHO classification of Tumours of the Digestive System (2010 version). Tumor stage was defined according to the American Joint Committee on Cancer/International Union Against Cancer TNM (tumor-node-metastasis) classification system (2010 version). The patients were followed every 3 month for the first year and then every 6 months for the next 2 years and finally annually after surgery. The tumor recurrence (including local recurrence or metastasis) was detected by ultrasonography, CT or MRI. The time of detection of recurrence was still not known until the patient was dead of ESCC, and the time to death was used instead.

\section{Pathological Evaluation}

Patient records and original histopathologic slides were independently reviewed by 2 pathologists with special experience in gastrointestinal pathology (S.-Y. Xi and M.-Y. Cai) who were blinded to the pathological diagnoses and outcome data. Discrepancies were solved by simultaneous re-examination of the slides by both pathologists with a double-headed microscope. A mean of 4.2 (median 4, range 3-8) paraffin-embedded tissue blocks per tumor were available for evaluation, and all of these patients had at least 3 tissue blocks available.

The presence of vascular invasion was carefully evaluated on hematoxylin and eosin (H\&E)-stained slides. Vascular invasion was defined as infiltration of vessel walls or the existence of tumor emboli [4]. The lymphatic channels were included in our study. Special care was taken to differentiate endothelial cells from retraction artifacts lined by fibroblasts.

\section{Statistical Analysis}

The correlation between vascular invasion and the clinicopathologic features of the ESCC patients was evaluated by a $\chi 2$-test. For univariate analysis, survival curves were obtained with the Kaplan-Meier method, and the differences between groups in survival were tested by the log-rank test. Multivariate survival analyses were performed with the Cox proportional hazard regression model. The Harrell concordance index (C-index) was employed to assess model prognostic accuracy on multivariate analysis. A significant difference was deemed if the $P$ value from a two-tailed test was less than 0.05. Statistical analysis was performed with SPSS statistical software package (SPSS Standard version 13.0; SPSS, Chicago, IL, USA) and R, version 3.0.1 (http://www.r-project.org/).

\section{Results}

\section{The Patterns of Vascular Invasion}

Vascular invasion in ESCC was identified as infiltration of vessel walls (Figure 1A) or the existence of tumor emboli in vascular spaces (Figure 1B). In the training cohort, presence of vascular invasion was observed in 113 of 216 (52.3\%) of ESCGs. Further correlation analysis demonstrated that the presence of vascular invasion was significantly correlated with tumor location, infiltrative depth, $\mathrm{pN}$ classification and stage in $\operatorname{ESCG}(P<0.05$, Table 1).

In the validation cohort, the presence of vascular invasion was found in 96 of 217 (44.2\%) of ESCGs. Similar to the observations in the training cohort, presence of vascular invasion was correlated closely to certain clinicopathological features, including patient age, $\mathrm{pN}$ classification and stage $(P<0.05$, Table 1$)$.

\section{Survival Analysis}

Assessment of survival in the training cohort of ESCG patients revealed that some clinicopathological parameters indicated a significant impact of prognosis, such as infiltrative depth $(P$ $=0.005), \mathrm{pN}$ classification $(P<0.0001)$, tumor stage $(P<0.0001)$ and vascular invasion $(P<0.0001$, Table 2$)$. The result demonstrated that the patients with vascular invasion displayed a poor overall survival (Table 2; Figure 2A) and recurrence-free survival (Figure 2B) than the patients without vascular invasion $(P<$ $0.0001)$. 


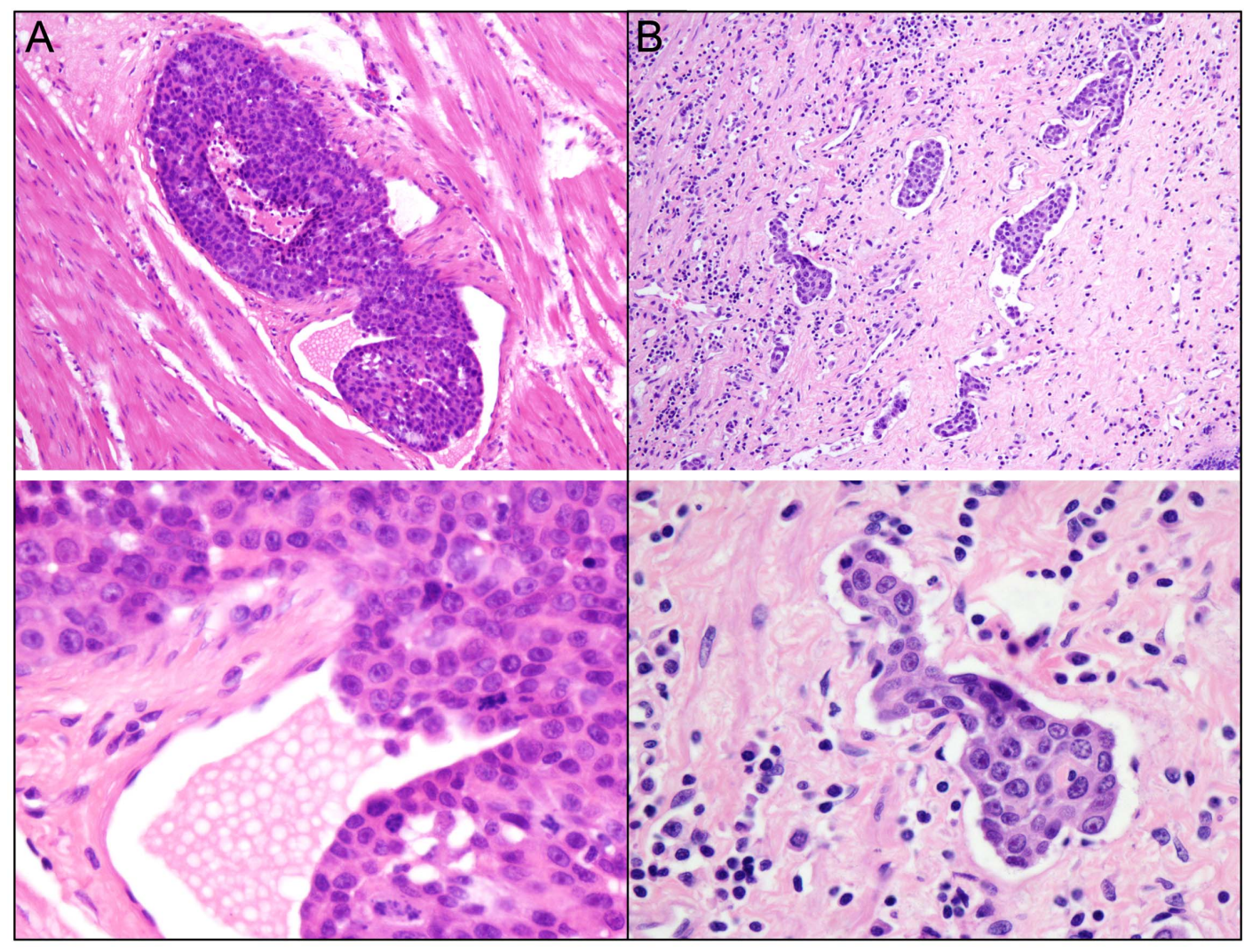

Figure 1. The patterns of vascular invasion in ESCC. A: Tumor cells were encroaching into the blood vessel wall (upper panel, $\times 100$; lower panel, $\times 400$ ). B: Tumor emboli were found in vascular spaces (upper panel, $\times 100$; lower panel, $\times 400$ ). doi:10.1371/journal.pone.0096129.g001

Results in the validation cohort were similar to those in the training cohort. Patients with presence of vascular invasion also showed a significant trend toward worse overall survival (Table 2; Figure 2C) and recurrence-free survival (Figure 2D) compared to the patients with absence of vascular invasion $(P<0.0001)$. Of the other prognostic factors, univariate analysis showed that gender $(P<0.05)$, differentiation $(P<0.05)$, lymph node status $(P<0.0001)$ and tumor stage $(P<0.0001)$ adversely affected patient diseasespecific survival (Table 2).

Further survival analysis was performed with regard to vascular invasion in the subset of ESCC patients without lymph node metastasis. Our results demonstrated that the presence of vascular invasion was identified as a prognostic predictor of overall survival and recurrence-free survival in ESCG patients without lymph node metastasis in both cohorts $(P<0.05$, Figure 3$)$.

\section{Multivariate Cox Regression Analysis on the Two Cohorts}

Since variables examined to have prognostic influence by univariate analysis may covariate, the presence of vascular invasion as well as other clinicopathologic features (including tumor size, differentiation, infiltrative depth, $\mathrm{pN}$ status and stage) were tested in multivariate analysis (Table 3). In the training cohort, the presence of vascular invasion was found to be a significantly independent prognostic factor for poor overall survival (hazard ratio, 1.622; 95\% CI, 1.096-2.401; $P=0.016$; Table 3). Similar results were also observed in our validation cohort (hazard ratio, 1.655; 95\% CI, 1.100-2.489; $P=0.016$; Table 3). Of the other parameters, $\mathrm{pN}$ classification was evaluated as an independent prognostic factor for patient survival in both cohorts.

\section{New Prognostic Model with pN Classification Supplemented by Vascular Invasion}

According to the results of our multivariate analyses, we proposed a new clinicopathologic prognostic model with 2 prognostic factors, i.e., $\mathrm{pN}$ classification and vascular invasion. We designated a high-risk group as the presence of the advanced $\mathrm{pN}$ classification (2 or 3 ) and vascular invasion, an intermediaterisk group as the presence of one factor [the presence of the advanced $\mathrm{pN}$ classification (2 or 3 ) or vascular invasion], and a low-risk group as the presence of none [the low $\mathrm{pN}$ classification (i.e., $\mathrm{pN} 0$ or $\mathrm{pNl}$ ) and absence of vascular invasion]. Our results revealed that the proposed model could significantly stratify the risk (low, intermediate and high) for overall survival (Figure $4 \mathrm{~A}$ and $4 \mathrm{~B}, P<0.0001$ ) and recurrence-free survival (Figure $4 \mathrm{C}$ and $4 \mathrm{D}, P<0.0001)$ in both cohorts.

In training cohort, application of $\mathrm{C}$-index to the proposed new prognostic model showed improved predictive ability when compared with the single $\mathrm{pN}$ classification model (c indexes of 0.785 vs 0.739 , respectively). Similar finding was observed in the validation cohort, the $\mathrm{C}$-index of the $\mathrm{pN}$ classification supplemented by vascular invasion was 0.689 compared to 0.650 in our validation study of the $\mathrm{pN}$ classification model.

\section{Discussion}

It has been suggested that the 5 -year overall survival of ESCG is 15-34\% [2-3] and suitable and individual management of ESCC is needed to improve the outcome for patients. TNM stage is traditionally considered the single most important prognostic factor of ESCC. Other features have been found to be prognostic 


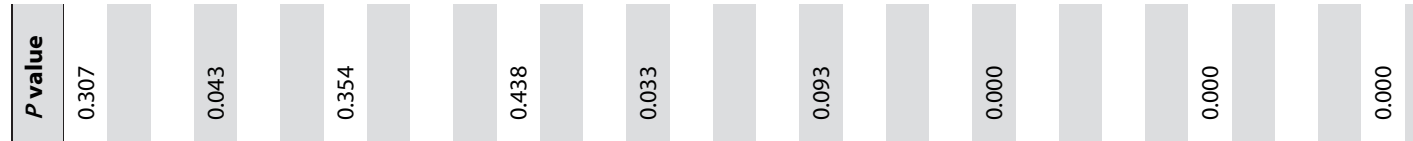

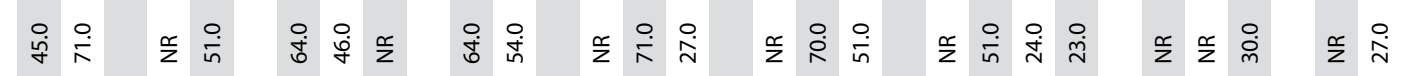

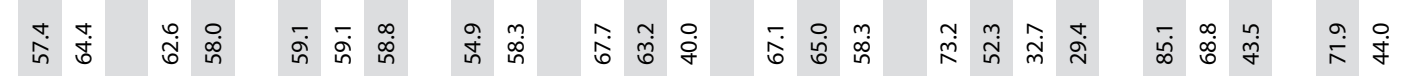

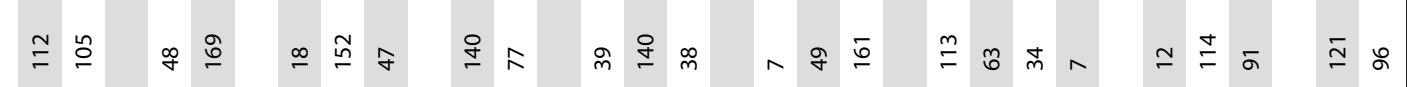

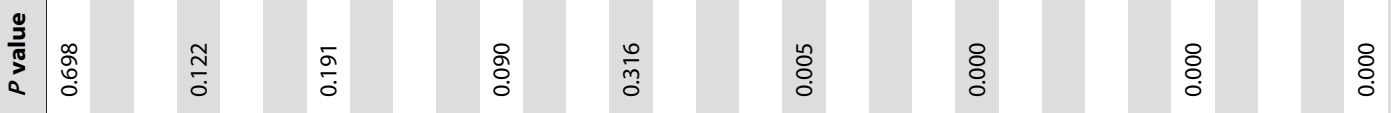


A

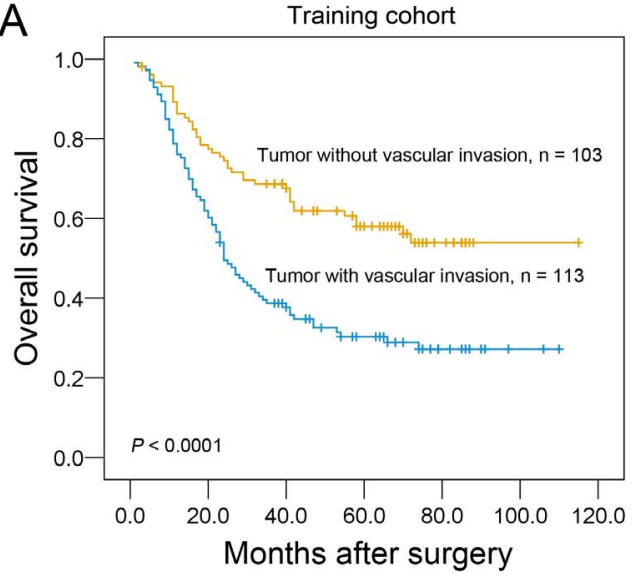

C

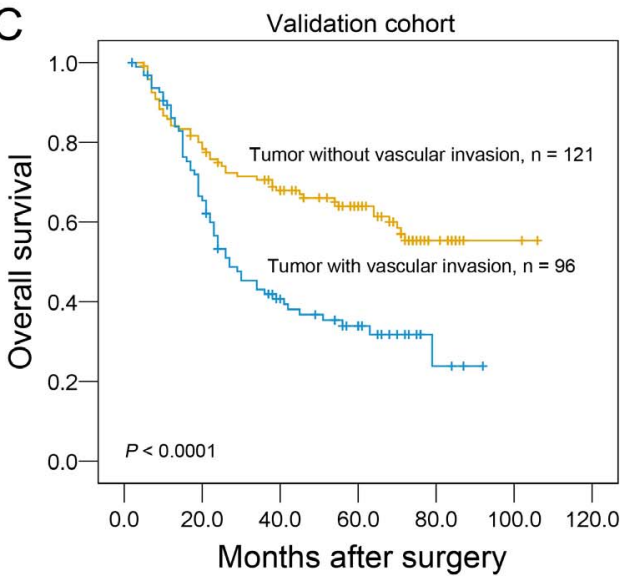

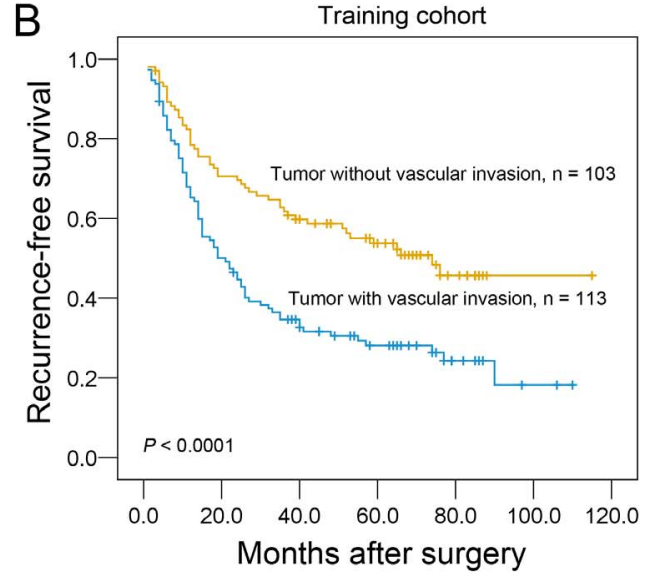

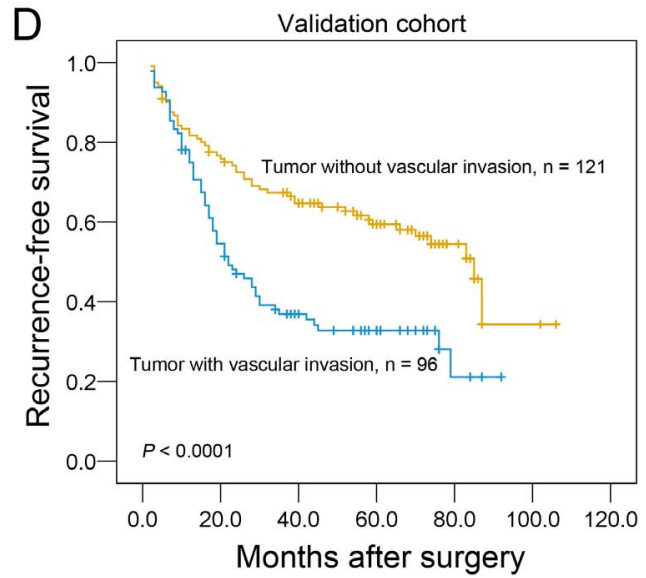

Figure 2. The association of vascular invasion with ESCC patients' survival (log-rank test). Kaplan-Meier survival analysis of vascular invasion for overall survival (A) and recurrence-free survival (B) in the training cohort. Kaplan-Meier survival analysis of vascular invasion for overall survival (C) and recurrence-free survival (D) in the validation cohort.

doi:10.1371/journal.pone.0096129.g002

assessment of patients with ESCC. In this regard, the presence of tumor size, differentiation, location, performance status of patient, tumor infiltration depth, lymph node status and distant metastasis have a major role, and are extensively utilized in clinical setting $[4,10-11,14,17-23]$.

In the current study, we assessed a retrospective collection of data on patients with ESCC to determine the prognostic accuracy of the $\mathrm{pN}$ classification compared to this classification supplemented by vascular invasion. Our results demonstrated that in testing and validation cohorts, the presence of vascular invasion was frequently observed in ESCC as evaluated on H\&E-stained slides. Further correlation analyses in testing cohort revealed that the presence of vascular invasion in ESCCs was significantly associated with tumor location, infiltrative depth, $\mathrm{pN}$ status and stage. Similar result was confirmed in our validation cohort. In addition, multivariate analyses in both cohorts evaluated that the presence of vascular invasion was a prognostic factor independent of certain well-established clinical factors, including tumor size, differentiation, $\mathrm{pT}$ status, $\mathrm{pN}$ status and clinical stage. The $\mathrm{G}-$ index analysis showed that the proposed new prognostic model (combined $\mathrm{pN}$ classification and vascular invasion) could improve the predictive ability when compared to $\mathrm{pN}$ classification.

Our findings of vascular invasion status and its correlation with ESCC patients' outcome are consistent with the results of other groups. In 1986, Sugimachi et al found a significant association between the presence of vascular invasion and 5-year survival as evidenced by univariate analysis [10]. A similar result was reported by Ide et al. [11], in which multivariate analysis of ESCC patients revealed that vascular invasion was a significant prognostic predictor of the overall survival. In a more recent study, Kitagawa et al. investigated the prognostic value of epidermal growth factor receptor (EGFR) gene amplification in patients with ESCG and found that vascular invasion was proved to retain independent prognostic value [12]. Importantly, vascular invasion in superficial ESCC is also found to be a strong independent predictor of lymph node metastasis with an odds ratio of 12.01 [14]. Notably other published reports show no significantly prognostic value for vascular invasion to predict the outcome in patients with ESCC [15-16]. Taken together, differences in clinicopathologic characteristics among cohorts, geographic backgrounds, methodology in vascular invasion detection, patient heterogeneity, lack of independent validation of the results, small sample size and different definitions of end points (disease-free, cancer specific or overall survival) might contribute to the controversial results.

Generally, our findings support the idea that the $\mathrm{pN}$ classification supplemented by vascular invasion might improve the ability to discriminate ESCC patients' outcome, especially in the patients without lymph node metastasis. As it is well known that the pTNM stage and tumor differentiation are the best-established risk factors for important aspects affecting the prognosis of patients with 

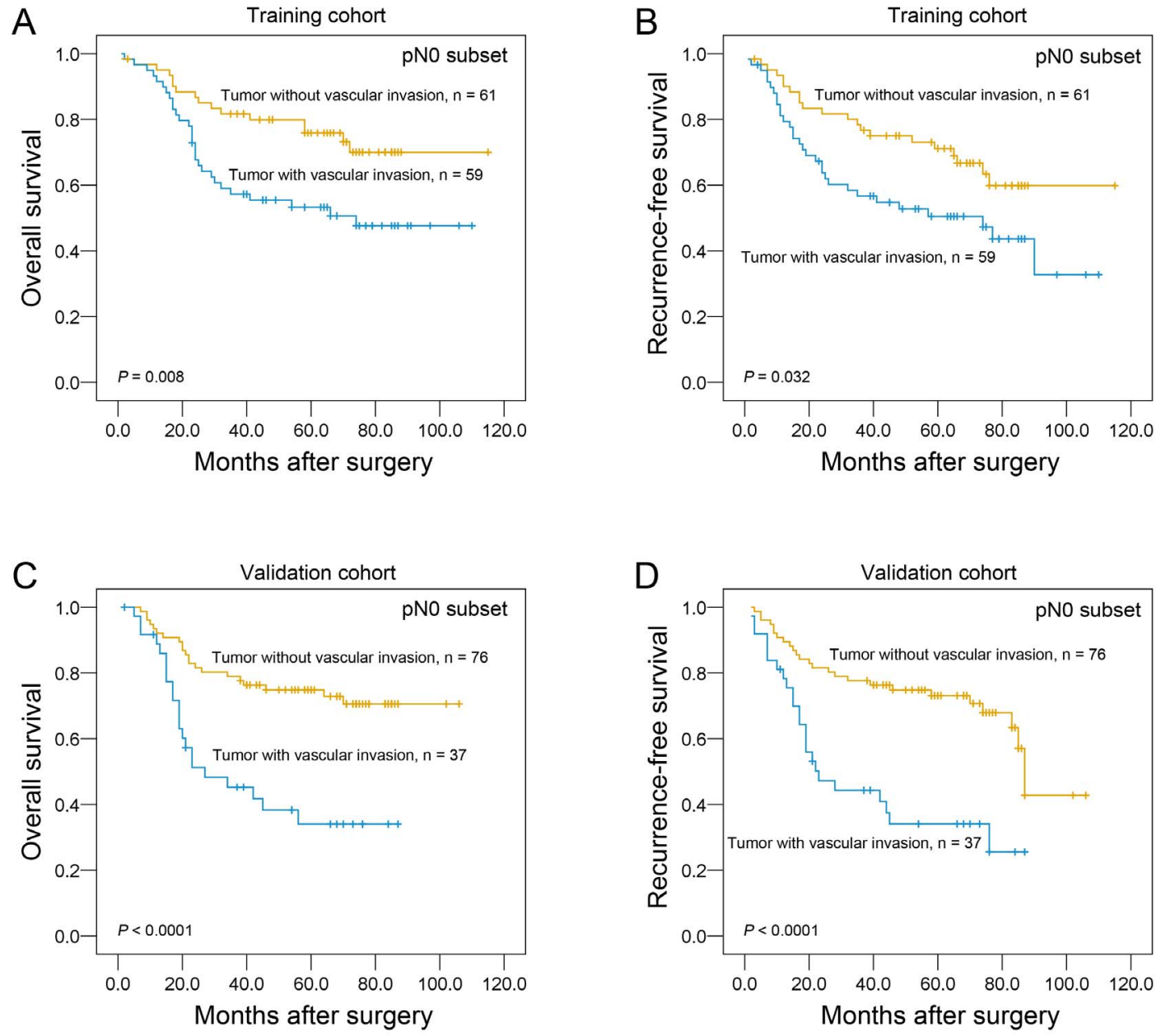

Figure 3. Univariate survival analysis with regard to vascular invasion in the subset of ESCC patients with pNO classification. The presence of vascular invasion was identified as a prognostic predictor of overall survival and recurrence-free survival in ESCC patients without lymph node metastasis in training cohort $(\mathrm{A}$ and $\mathrm{B})$ and validation cohort ( $\mathrm{C}$ and $\mathrm{D})$.

doi:10.1371/journal.pone.0096129.g003

Table 3. Cox multivariate analyses of prognostic factors for overall survival.

\begin{tabular}{|c|c|c|c|}
\hline Variables & Hazards ratio & $95 \% \mathrm{Cl}$ & $P$ value \\
\hline \multicolumn{4}{|l|}{ Training cohort } \\
\hline Tumor size, $\mathrm{cm}\left(\leq 4^{*} v>4\right)$ & 1.134 & $0.783-1.643$ & 0.506 \\
\hline Differentiation (well $v$ moderate $v$ poor) & 1.103 & $0.816-1.490$ & 0.525 \\
\hline pT classification (T1 $v$ T2 $v$ T3) & 1.113 & $0.726-1.706$ & 0.622 \\
\hline pN classification (N0 $v$ N1 $v$ N2 $v$ N3) & 1.759 & $1.260-2.455$ & 0.001 \\
\hline Stage $(|v \|||v|||)$ & 1.505 & $0.771-2.937$ & 0.231 \\
\hline Vascular invasion (absent $v$ present) & 1.622 & $1.096-2.401$ & 0.016 \\
\hline \multicolumn{4}{|l|}{ Validation cohort } \\
\hline Tumor size, $\mathrm{cm}(\leq 4 v>4)$ & 1.104 & $0.747-1.633$ & 0.619 \\
\hline Differentiation (well $v$ moderate $v$ poor) & 1.181 & $0.853-1.634$ & 0.317 \\
\hline pT classification (T1 $v$ T2 $v$ T3) & 1.421 & $0.900-2.241$ & 0.131 \\
\hline pN classification (N0 $v$ N1 $v$ N2 $v$ N3) & 1.512 & $1.087-2.103$ & 0.014 \\
\hline Stage (I $v\|v\|$ III) & 0.924 & $0.513-1.663$ & 0.791 \\
\hline Vascular invasion (absent $v$ present) & 1.655 & $1.100-2.489$ & 0.016 \\
\hline
\end{tabular}

*Median size; $\mathrm{Cl}$, confidence interval.

doi:10.1371/journal.pone.0096129.t003 
A

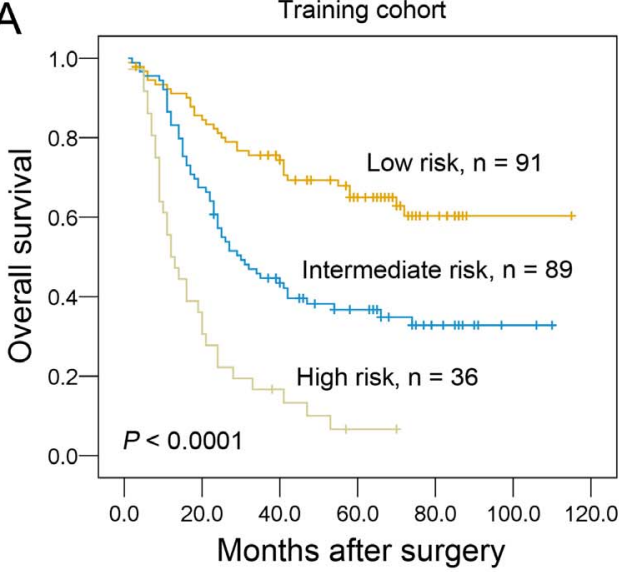

$\mathrm{C}$

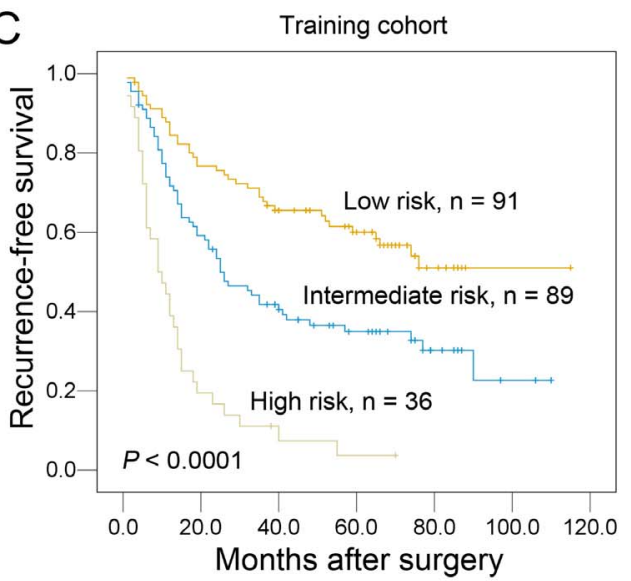

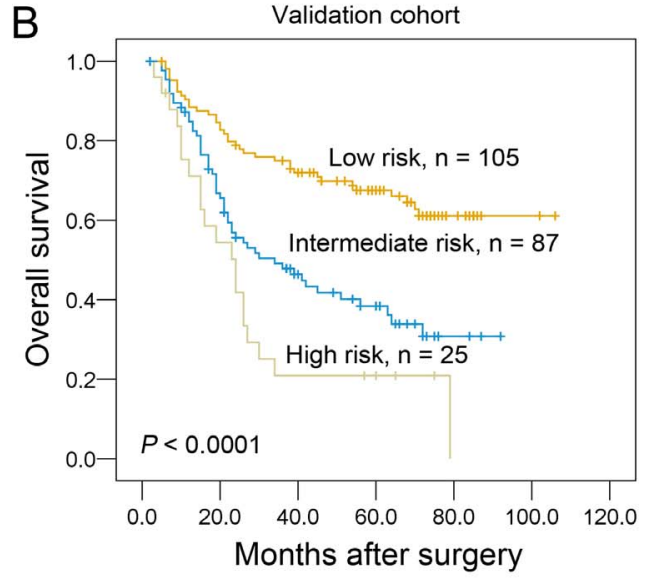

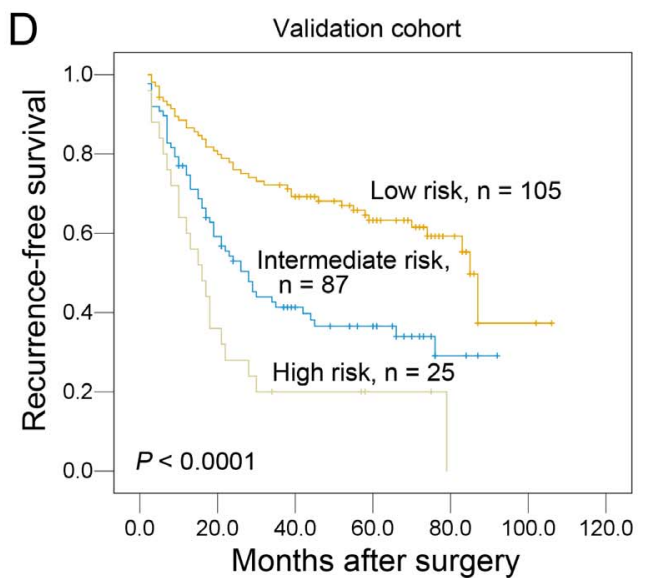

Figure 4. Comparison of overall survival according to a new combined prognostic model. The new combined model (including pN classification and vascular invasion) could significantly stratify the risk (low, intermediate and high) for overall survival of ESCC patients in training cohort (A) and validation cohort (B). The proposed model could significantly discriminate the risk (low, intermediate and high) for recurrence-free survival of ESCC patients in training cohort (C) and validation cohort (D). doi:10.1371/journal.pone.0096129.g004

ESCC. These two variables, based on specific clinicopathologic features and extent of disease, may have reached their limits in providing critical information influencing patient prognosis and treatment strategies. Furthermore, outcome of patients with same stage following surgery is substantially different and such large discrepancy has not been well understood [24-25]. Thus, there is a need for new objective strategies that can effectively distinguish between patients with favorable and unfavorable outcome. In the present study, our data support the concept that vascular invasion, as detected by H\&E staining, can identify ESCG patients with or without aggressive clinical course and/or poor outcome. Thus, evaluation of vascular invasion may become a factor for predicting prognosis and rendering a more tailored treatment strategy in patients with ESCG. Based on our results, we proposed a new prognostic model with $\mathrm{pN}$ classification supplemented by vascular invasion. This combined model can reflect the aggressive phenotype of ESCC. There are also strong efforts to integrate biomarkers into established clinicopathologic models to further improve their predictive ability [26]. Thus, this combined model may be a useful prognostic index for ESCC.

Although the present study was retrospective, it was strengthened by the fact that all histopathologic slides were reviewed by two gastrointestinal pathologists. Moreover, all classical clinicopathologic features were re-evaluated and compared to vascular invasion, which stood out as the most relevant predictor of ESCC aggressiveness. At last, we proposed a new prognostic model combining $\mathrm{pN}$ classification with vascular invasion that could be easily determined by the pathologists and used to accurately predict the biological behavior of ESCG. To our knowledge, this is the first report to investigate the prognostic ability of the $\mathrm{pN}$ classification supplemented by vascular invasion; however, further external validation of this important model is needed using pooled multicenter data.

In the present study, we observed that presence of vascular invasion was a strong and independent predictor of adverse survival, as evidenced by Kaplan-Meier curves and multivariate Cox proportional hazard regression analysis. The proposed new prognostic model with the $\mathrm{pN}$ classification supplemented by vascular invasion might improve the ability to discriminate ESCC patients' outcome. Thus, the examination of vascular invasion could be used as an additional effective instrument in identifying those ESCC patients at increased risk of tumor progression. This instrument might also help the clinician to choose a suitable therapy for the individual patient, for example, favoring a more aggressive treatment in patients with vascular invasion. 


\section{Author Contributions}

Conceived and designed the experiments: MYC. Performed the experiments: CMZ YHL. Analyzed the data: SYX RZL JWC. Contributed

\section{References}

1. Jemal A, Murray T, Ward E, Samuels A, Tiwari RC, et al. (2005) Cancer statistics, 2005. CA Cancer J Clin 55: 10-30.

2. Allum WH, Stenning SP, Bancewicz J, Clark PI, Langley RE (2009) Long-term results of a randomized trial of surgery with or without preoperative chemotherapy in esophageal cancer. J Clin Oncol 27: 5062-5067.

3. Sjoquist KM, Burmeister BH, Smithers BM, Zalcberg JR, Simes RJ, et al. (2011) Survival after neoadjuvant chemotherapy or chemoradiotherapy for resectable oesophageal carcinoma: an updated meta-analysis. Lancet Oncol 12: 681-692.

4. Li H, Zhang Q, Xu L, Chen Y, Wei Y, et al. (2009) Factors predictive of prognosis after esophagectomy for squamous cell cancer. J Thorac Cardiovasc Surg 137: 55-59.

5. Rice TW, Blackstone EH, Rusch VW (2010) 7th edition of the AJCC Cancer Staging Manual: esophagus and esophagogastric junction. Ann Surg Oncol 17: 1721-1724.

6. Sobin LH, Fleming ID (1997) TNM Classification of Malignant Tumors, fifth edition (1997). Union Internationale Contre le Cancer and the American Joint Committee on Cancer. Cancer 80: 1803-1804.

7. Compton CC, Fielding LP, Burgart LJ, Conley B, Cooper HS, et al. (2000) Prognostic factors in colorectal cancer. College of American Pathologists Consensus Statement 1999. Arch Pathol Lab Med 124: 979-994.

8. Fisseler-Eckhoff A (2009) [New TNM classification of malignant lung tumors 2009 from a pathology perspective]. Pathologe 30 Suppl 2: 193-199.

9. Kojima M, Shimazaki H, Iwaya K, Kage M, Akiba J, et al. (2013) Pathological diagnostic criterion of blood and lymphatic vessel invasion in colorectal cancer: a framework for developing an objective pathological diagnostic system using the Delphi method, from the Pathology Working Group of the Japanese Society for Cancer of the Colon and Rectum. J Clin Pathol 66: 551-558.

10. Sugimachi K, Matsuura H, Kai H, Kanematsu T, Inokuchi K, et al. (1986) Prognostic factors of esophageal carcinoma: univariate and multivariate analyses. J Surg Oncol 31: 108-112.

11. Ide H, Nakamura T, Hayashi K, Endo T, Kobayashi A, et al. (1994) Esophageal squamous cell carcinoma: pathology and prognosis. World J Surg 18: 321-330.

12. Kitagawa Y, Ueda M, Ando N, Ozawa S, Shimizu N, et al. (1996) Further evidence for prognostic significance of epidermal growth factor receptor gene amplification in patients with esophageal squamous cell carcinoma. Clin Cancer Res 2: 909-914.

13. Torres CM, Wang HH, Turner JR, Richards W, Sugarbaker D, et al. (1999) Pathologic prognostic factors in esophageal squamous cell carcinoma: a followup study of 74 patients with or without preoperative chemoradiation therapy. Mod Pathol 12: 961-968.

14. Mitobe J, Ikegami M, Urashima M, Takahashi H, Goda K, et al. (2013) Clinicopathological investigation of lymph node metastasis predictors in reagents/materials/analysis tools: JPY DX. Wrote the paper: CMZ YHL MYC.

superficial esophageal squamous cell carcinoma with a focus on evaluation of lympho-vascular invasion. Scand J Gastroenterol 48: 1173-1182.

15. Sliwa B, Szelachowski P, Strutynska-Karpinska M (2013) An analysis of the impact of clinico-pathological features on long-term results following esophagectomy due to squamous cell carcinoma of the thoracic esophagus. Adv Clin Exp Med 22: 369-375.

16. Zhu Z, Yu W, Li H, Zhao K, Zhao W, et al. (2013) Nodal skip metastasis is not a predictor of survival in thoracic esophageal squamous cell carcinoma. Ann Surg Oncol 20: 3052-3058.

17. Rahman AA, Singh R, Sharma P (2013) Management of Barrett's esophagus. Minerva Gastroenterol Dietol 59: 13-23.

18. Ren HZ, Wang JS, Wang P, Pan GQ, Wen JF, et al. (2010) Increased expression of prohibitin and its relationship with poor prognosis in esophageal squamous cell carcinoma. Pathol Oncol Res 16: 515-522.

19. Sgourakis G, Gockel I, Lyros O, Lanitis S, Dedemadi G, et al. (2012) The use of neural networks in identifying risk factors for lymph node metastasis and recommending management of tlb esophageal cancer. Am Surg 78: 195-206.

20. Robey-Cafferty SS, el-Naggar AK, Sahin AA, Bruner JM, Ro JY, et al. (1991) Prognostic factors in esophageal squamous carcinoma. A study of histologic features, blood group expression, and DNA ploidy. Am J Clin Pathol 95: 844 849.

21. Sarbia M, Porschen R, Borchard F, Horstmann O, Willers R, et al. (1995) Incidence and prognostic significance of vascular and neural invasion in squamous cell carcinomas of the esophagus. Int J Cancer 61: 333-336.

22. Waraich N, Rashid F, Jan A, Semararo D, Deb R, et al. (2011) Vascular invasion is not a risk factor in oesophageal cancer recurrence. Int J Surg 9: 237240.

23. Inoue A, Moriya H, Katada N, Tanabe S, Kobayashi N, et al. (2008) Intratumoral lymphangiogenesis of esophageal squamous cell carcinoma and relationship with regulatory factors and prognosis. Pathology International 58: 611-619.

24. Nishimaki T, Suzuki T, Suzuki S, Kuwabara S, Hatakeyama K (1998) Outcomes of extended radical esophagectomy for thoracic esophageal cancer. J Am Coll Surg 186: 306-312.

25. Fujita H, Kakegawa T, Yamana H, Shima I, Toh Y, et al. (1995) Mortality and morbidity rates, postoperative course, quality of life, and prognosis after extended radical lymphadenectomy for esophageal cancer. Comparison of threefield lymphadenectomy with two-field lymphadenectomy. Ann Surg 222: 654 662.

26. Pichler M, Hutterer GC, Chromecki TF, Jesche J, Groselj-Strele A, et al. (2012) Prognostic value of the Leibovich prognosis score supplemented by vascular invasion for clear cell renal cell carcinoma. J Urol 187: 834-839. 